\title{
Alectinib-induced Immune Hemolytic Anemia in a Patient with Lung Adenocarcinoma
}

\author{
Joe Okumoto ${ }^{1}$, Shinjiro Sakamoto ${ }^{1}$, Takeshi Masuda ${ }^{1}$, Kakuhiro Yamaguchi ${ }^{1}$, \\ Yasushi Horimasu ${ }^{1}$, Shintaro Miyamoto ${ }^{1}$, Taku Nakashima ${ }^{1}$, Hiroshi Iwamoto ${ }^{1}$, \\ Noriyasu Fukushima ${ }^{2}$, Kazunori Fujitaka ${ }^{1}$, Hironobu Hamada ${ }^{1}$ and Noboru Hattori ${ }^{1}$
}

\begin{abstract}
:
Drug-induced immune hemolytic anemia (DIIHA) is a rare condition with an increasing incidence associated with the frequent use of certain drugs. An 85-year-old woman with lung adenocarcinoma prescribed alectinib complained of dyspnea on exertion at our hospital. Based on her laboratory tests results on admission, we focused on the clinical course of anemia and hemolysis progression after alectinib administration. The patient's anemia and hemolysis gradually improved after discontinuation of alectinib, leading to a diagnosis of alectinib-induced IHA, presented here as the first case encountered in a patient with lung adenocarcinoma. Furthermore, we discuss the importance of correlating clinical laboratory findings in DIIHA.
\end{abstract}

Key words: alectinib, drug-induced immune hemolytic anemia, lung cancer, anaplastic lymphoma kinase, coombs-negative autoimmune hemolytic anemia

(Intern Med 60: 611-615, 2021)

(DOI: 10.2169/internalmedicine.4241-19)

\section{Introduction}

The incidence of drug-induced immune hemolytic anemia (DIIHA) is approximately 1 per million yearly (1). Despite its rare occurrence, there are reports of DIIHA caused by frequently used drugs. Thus, the possibility of DIIHA should be considered in connection with their use.

Recently, several drugs against various molecular targets have been developed, resulting in a remarkable improvement in the prognosis of lung cancer harboring a driver mutation. An epidermal growth factor receptor $(E G F R)$ gene mutation and the anaplastic lymphoma kinase $(A L K)$ fusion gene are representative driver mutations in lung cancer and targets of molecular-targeted drugs (2).

ALK-positive lung cancer accounts for approximately $4 \%$ of all lung cancer cases (2). ALK domain-targeted tyrosine kinase inhibitors (ALK-TKIs) are being rapidly developed (3). Crizotinib is a first-generation ALK-TKI, whereas alectinib and ceritinib are second-generation and lorlatinib is a third-generation drug. Guidelines recommend alectinib as the primary therapy for ALK-positive lung cancer, making it a key drug for the treatment of this condition.

Anemia accounts for $5-15 \%$ of the adverse events associated with alectinib (4). However, to our knowledge, alectinib-induced immune hemolytic anemia (IHA) has not been reported before. We herein report the first case of alectinib-induced IHA in a patient with lung adenocarcinoma. Furthermore, in this case, the direct Coombs' test [also called the direct antiglobulin test (DAT)] was negative. We therefore also discuss the characteristic findings of patients with DAT-negative autoimmune hemolytic anemia (AIHA).

\section{Case Report}

An 85-year-old woman primarily presenting with dyspnea on exertion visited a local hospital in December 2016. Chest X-ray showed left pleural effusion, and she subsequently visited our hospital for a detailed examination. Chest com-

${ }^{1}$ Department of Molecular and Internal Medicine, Hiroshima University Hospital, Japan and ${ }^{2}$ Department of Hematology, Hiroshima University Hospital, Japan

Received: November 22, 2019; Accepted: August 16, 2020; Advance Publication by J-STAGE: September 30, 2020

Correspondence to Dr. Shinjiro Sakamoto, s-sakamoto@hiroshima-u.ac.jp 
Table 1. Laboratory Data.

\begin{tabular}{lcllll}
\hline <Hematology $>$ & \multicolumn{5}{l}{$<$ Biochemistry $>$} \\
WBC & $5,710 / \mu \mathrm{L}$ & T-Bil & $2.6 \mathrm{mg} / \mathrm{dL}$ & $\mathrm{TP}$ & $6.4 \mathrm{~g} / \mathrm{dL}$ \\
$\mathrm{RBC}$ & $2.12 \times 10^{6} / \mu \mathrm{L}$ & $\mathrm{D}-\mathrm{Bil}$ & $0.8 \mathrm{mg} / \mathrm{dL}$ & $\mathrm{Alb}$ & $3.4 \mathrm{~g} / \mathrm{dL}$ \\
$\mathrm{Hb}$ & $7.3 \mathrm{~g} / \mathrm{dL}$ & $\mathrm{AST}$ & $35 \mathrm{U} / \mathrm{L}$ & $\mathrm{Fe}$ & $85 \mu \mathrm{g} / \mathrm{dL}$ \\
$\mathrm{Hct}$ & $23.6 \%$ & $\mathrm{ALT}$ & $21 \mathrm{U} / \mathrm{L}$ & $\mathrm{UIBC}$ & $193 \mu \mathrm{g} / \mathrm{dL}$ \\
$\mathrm{MCV}$ & $111.3 \mathrm{fl}$ & $\mathrm{LDH}$ & $281 \mathrm{U} / \mathrm{L}$ & Ferritin & $17.7 \mathrm{ng} / \mathrm{mL}$ \\
$\mathrm{MCH}$ & $34.4 \mathrm{pg}$ & $\mathrm{ALP}$ & $488 \mathrm{U} / \mathrm{L}$ & Folic Acid & $5.2 \mathrm{ng} / \mathrm{mL}$ \\
$\mathrm{MCHC}$ & $30.9 \%$ & $\gamma-\mathrm{GTP}$ & $45 \mathrm{U} / \mathrm{L}$ & Vit-B12 & $1,757 \mathrm{pg} / \mathrm{mL}$ \\
$\mathrm{Plt}$ & $253 \times 10^{3} / \mu \mathrm{L}$ & $\mathrm{BUN}$ & $36.1 \mathrm{mg} / \mathrm{dL}$ & Haptoglobin & $<10 \mathrm{mg} / \mathrm{dL}$ \\
Ret & $3.64 \%$ & $\mathrm{Cr}$ & $1.63 \mathrm{mg} / \mathrm{dL}$ & $<$ Serology $>$ \\
$<$ Urinalysis $>$ & & $\mathrm{Na}$ & $139 \mathrm{mEq} / \mathrm{L}$ & $\mathrm{CRP}$ & $0.1 \mathrm{mg} / \mathrm{dL}$ \\
Specific gravity & 1.015 & $\mathrm{~K}$ & $5 \mathrm{mEq} / \mathrm{L}$ & $<$ Tumor marker $>$ \\
Proteinuria & $(-)$ & $\mathrm{Cl}$ & $109 \mathrm{mEq} / \mathrm{L}$ & CEA & $3.1 \mathrm{ng} / \mathrm{mL}$ \\
Hematuria & $(-)$ & & & $<$ Direct Coombs Test $>$ \\
Glucosuria & $(-)$ & & & negative & \\
\hline
\end{tabular}

WBC: white blood cells, RBC: red blood cells, Hb: hemoglobin, HCT: hematocrit, MCV: mean corpuscular volume, MCH: mean corpuscular hemoglobin, MCHC: mean corpuscular hemoglobin concentration, Plt: platelets, Ret: reticulocyte, T-Bil: total bilirubin, D-Bil: direct bilirubin, AST: asparatate aminotransferase, ALT: alanine aminotransferase, LDH: lactate dehydrogenase, ALP: alkaline phosphatase, $\gamma$-GTP: $\gamma$-glutamyl transpeptidase, BUN: blood urea nitrogen, Cr: creatinine, Na: sodium, K: potassium, $\mathrm{Cl}$ : chlorine, TP: total protein, Alb: albumin, Fe: serum iron, UIBC: unsaturated iron binding capacity, CRP: C-reactive protein, CEA: carcinoembryonic antigen

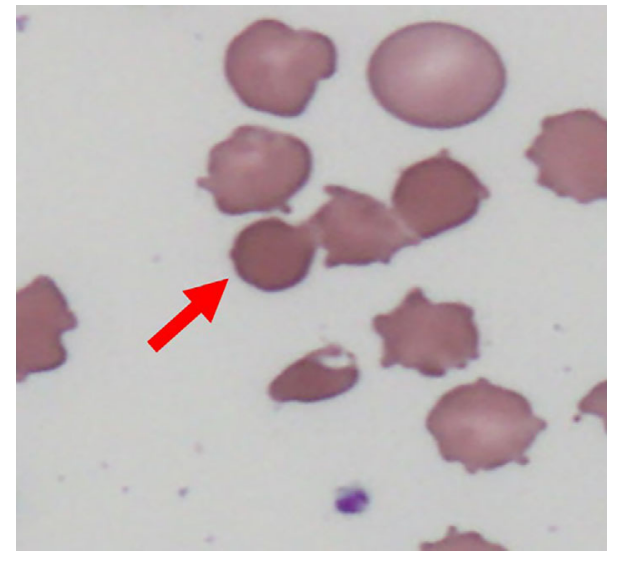

Figure 1. Spherocytes (arrow) were observed in peripheral blood smear on admission.

puted tomography (CT) revealed tumors in the left lower lobe of the lung and left pleural effusion. After a comprehensive examination, she was diagnosed with lung adenocarcinoma, specifically clinical T2aNOM1a stage IV without EGFR mutations. Accordingly, pemetrexed therapy was started as the first-line therapy in January 2017 but was discontinued after one cycle because of an adverse event. At that time, the patient tested positive for the $A L K$ fusion gene in the tissue obtained at a transbronchial biopsy. Consequently, alectinib therapy was started as the second-line therapy in February 2017, which induced a partial response and was subsequently continued.

However, the patient complained of dyspnea on exertion and visited our hospital in November 2018. Laboratory findings showed progressive anemia $(7.3 \mathrm{~g} / \mathrm{dL})$ and increased levels of total bilirubin (T-Bil, $2.6 \mathrm{mg} / \mathrm{dL}$ ), reticulocytes (3.64\%), and lactate dehydrogenase (LDH, $281 \mathrm{U} / \mathrm{L}$ ), whereas a low level of haptoglobin $(<10 \mathrm{mg} / \mathrm{dL})$ was reported (Table 1). The patient had macrocytic anemia but no history of stomach surgery, and her serum vitamin B12 and folic acid levels were normal. Furthermore, microspherocytes appeared on a peripheral blood smear (Fig. 1). In addition, the results for the direct Coombs' test (also called the DAT) were negative. We therefore conducted a CT examination to investigate the cause of hemolytic anemia.

Chest CT on admission showed no marked change in the primary tumor in the left $\mathrm{S}^{10}$ compared with chest CT performed six months earlier (Fig. 2). Conversely, abdominal CT on admission showed spleen enlargement compared with abdominal CT performed two years earlier. We therefore focused on the clinical course of anemia and hemolysis progression.

Because anemia and hemolysis progression were observed after the administration of alectinib (Fig. 3), we suspected alectinib-induced IHA. Consequently, we instructed the patient to discontinue alectinib and monitored the anemia over time. She needed a blood transfusion only once on hospital admission day 8 for dyspnea on exertion, but there was no further progression of anemia after the discontinuation of alectinib. Subsequently, the anemia and hemolysis gradually improved (Fig. 4). Therefore, she was confirmed to have had DAT-negative alectinib-induced IHA. In addition, the lung cancer has not worsened to date.

\section{Discussion}

We herein report the first case of alectinib-induced IHA 


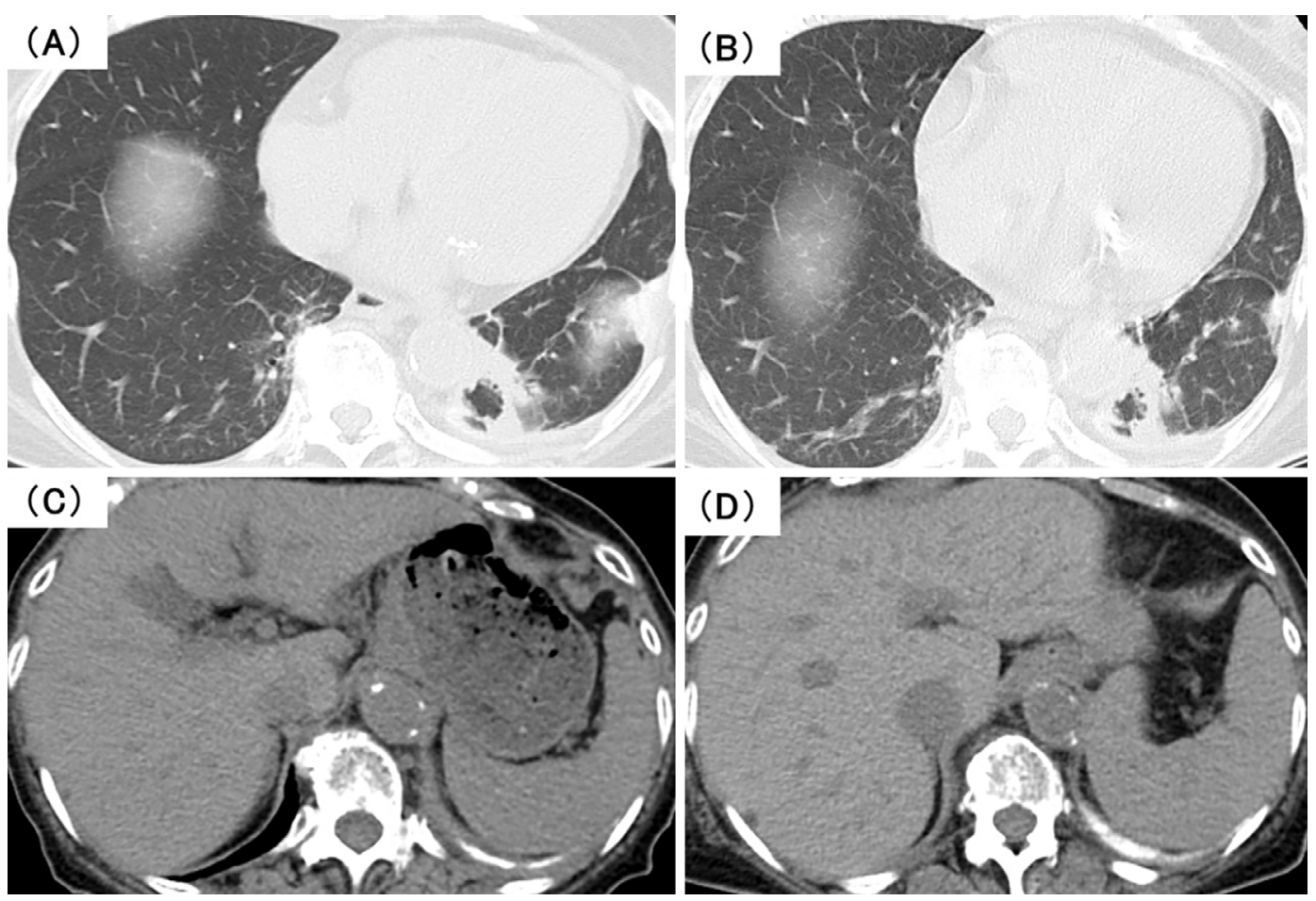

Figure 2. Chest computed tomography (CT) on admission. (A) Chest CT from six months earlier. (B) Chest CT six months later showing no marked change in the primary tumor in left $\mathrm{S}^{10}$ compared with (A). (C) Abdominal CT of spleen taken two years earlier. (D) Abdominal CT showing spleen enlargement compared with $(C)$. Dimensions, $78 \times 33 \times 70 \mathrm{~mm} \rightarrow 87 \times 41 \times 80 \mathrm{~mm}$.

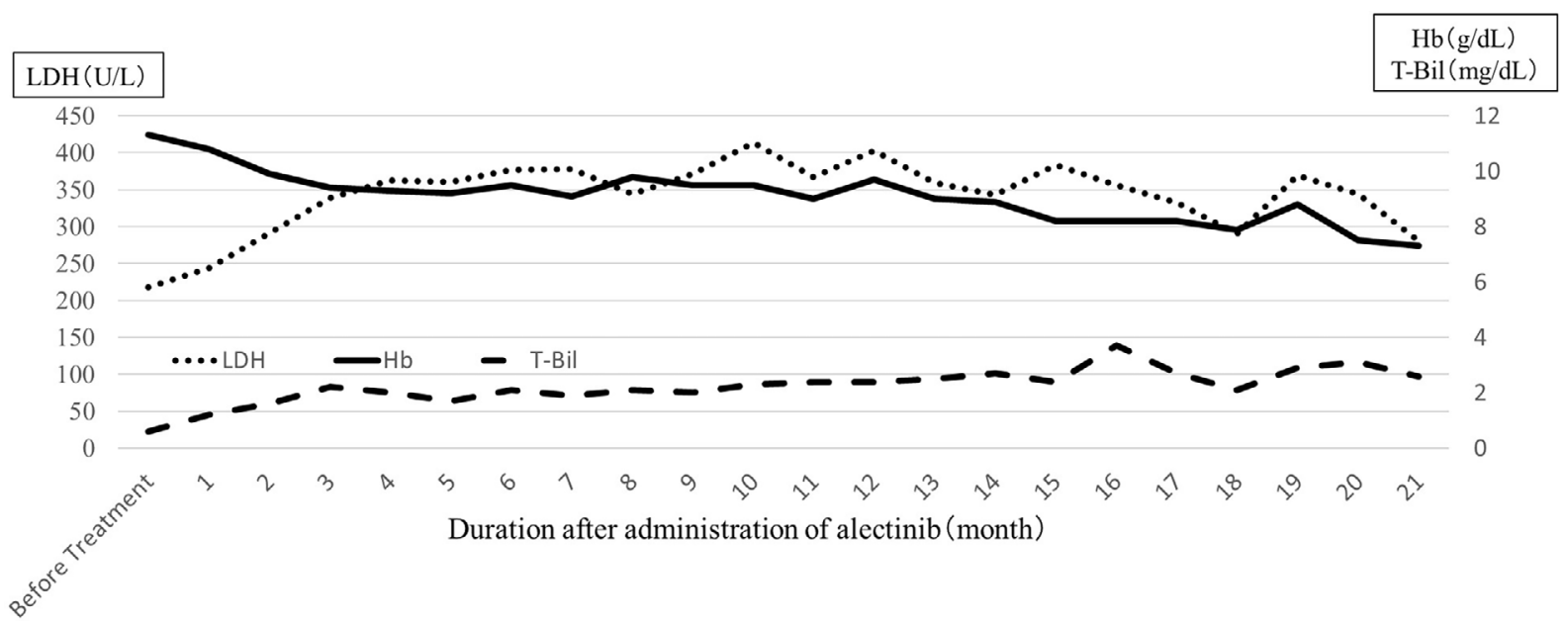

Figure 3. Changes in blood levels of hemoglobin, LDH, and T-Bil after the administration of alectinib. The hemoglobin level decreased, whereas the LDH and T-Bil levels gradually increased after alectinib administration. LDH: lactate dehydrogenase, T-Bil: total bilirubin

in a patient with lung adenocarcinoma. It has been reported that penicillin, acetaminophen, and alpha-methyldopa are frequent causes of DIIHA, but many other drugs can also be involved (5). DIIHA is classified according to three mechanisms of action: drug-absorption (hapten-induced), immune complex, or autoantibody (Table 2) (5). In this patient, prolonged drug administration, gradual progressive anemia, and extravascular hemolysis corresponded to the clinical features of the autoantibody type. In addition, the progression of splenomegaly after the administration of alectinib suggested extravascular hemolysis. Recently, immune checkpoint inhibitor (ICI)-induced IHA has been described in numerous case reports (6). The mechanism underlying ICI-induced IHA is considered to relate to the imbalance of regulatory $\mathrm{T}$ cells and the overactivity of $\mathrm{B}$ and $\mathrm{T}$ cells $(7,8)$. While this mechanism is assumed to be autoantibody type, it is still incompletely understood. In contrast, only case reports of hemolytic anemia with molecular-targeted agents, such as EGFR-TKIs and ALK-TKIs, have been published (9). TKIs are presumed to interfere with B-cell activation and the in- 


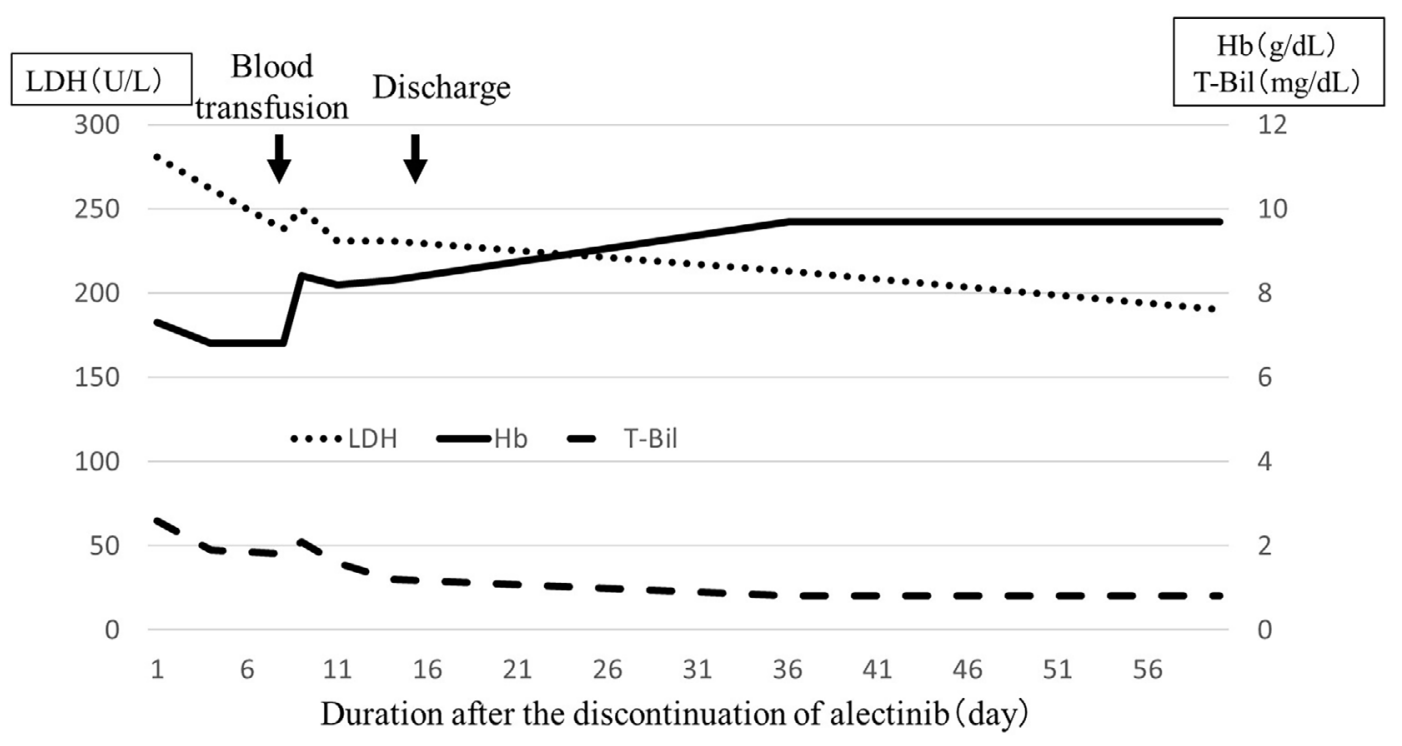

Figure 4. Changes in blood levels of hemoglobin, LDH, and T-Bil after the discontinuation of alectinib. The patient required blood transfusion only once on admission day 8 for dyspnea on exertion. The hemoglobin level then increased, while the LDH and T-Bil levels decreased gradually after alectinib discontinuation. LDH: lactate dehydrogenase, T-Bil: total bilirubin

Table 2. Three Mechanisms of Drug-induced Immune Hemolytic Anemia and This Case.

\begin{tabular}{lcccc}
\hline \multicolumn{1}{c}{ Mechanism } & Hapten-induced & Immune complex & Autoantibody & This case \\
\hline Medication & Penicillin & Acetaminophen & Alpha-methyldopa & Alectinib \\
& Cephalothin & Quinidine Rifampin & L-dopa & \\
Dose or Duration of drug & Large & Small & Large or Long-term & Long-term \\
Progress & Subacute & Acute & Chronic & Chronic \\
Site of hemolysis & Extravascular & Intravascular & Extravascular & Extravascular \\
Direct antiglobulin test & Positive & Positive & Positive & Negative \\
\hline
\end{tabular}

duction of the humoral immune response through their offtarget multikinase inhibitory effects (10), which may explain why these molecular-targeted agents carry a lower risk of DIIHA than cytotoxic anti-cancer agents and ICIs. In the present patient, first-line pemetrexed therapy may have contributed to the onset of alectinib-induced IHA. Cases of rheumatoid arthritis induced by pemetrexed therapy have been reported (11), and recent investigations have demonstrated that pemetrexed promotes lymphocyte activation (12). For this reason, first-line pemetrexed therapy probably induced autoantibody production and caused alectinibinduced IHA despite pemetrexed discontinuation. However, the characteristics of the molecular-targeted agents may have suppressed excessive autoantibody production and resulted in DAT-negative DIIHA rather than DAT-positive DIIHA.

DIIHA is caused by antibodies directed against antigens on the red blood cell (RBC) surface (13). Microspherocytes on a peripheral smear and a positive result for direct Coombs' test are the typical characteristic findings for the diagnosis of DIIHA (1). DIIHA has the same pathophysiology as AIHA, except that the cause is a drug. A direct Coombs' test distinguishes AIHA from hereditary spherocytosis (HS), paroxysmal nocturnal hemoglobinuria (PNH), hemolytic uremic syndrome (HUS), and thrombotic thrombocytopenic purpura (TTP) (14). However, the direct Coombs' test result in this case was negative. In addition, there were no schistocytes on the peripheral smear, no family history, and the laboratory data and symptoms negated a differential diagnosis, which led us to suspect DAT-negative DIIHA. Nevertheless, few reports have been published on DAT-negative DIIHA. Thus, in the following paragraph, we discuss DAT-negative AIHA instead of DAT-negative DIIHA.

DAT-negative AIHA, which accounts for approximately $10 \%$ of all AIHA cases (15), is principally attributable to (a) RBC-bound IgG below the threshold of detection using standard methods [ $>90 \%$ of cases (16)]; (b) a low affinity of IgG; and (c) RBC-bound IgA or IgM (17). When AIHA patients are DAT negative, evaluating RBC-bound $\mathrm{IgG}$ using an immunoradiometric method is recommended (17). In the present case, we were unable to evaluate RBC-bound IgG due to no sample being available. The degree of anemia and hemolysis is reported to be significantly milder in DATnegative AIHA than in DAT-positive AIHA (18). In the present patient, mild anemia, chronic clinical course, and immediate improvement of anemia after the discontinuation of 
alectinib corresponded to the clinical features of DATnegative AIHA. Based on these clinical and laboratory findings, the patient was diagnosed with alectinib-induced IHA.

In summary, we encountered a case of alectinib-induced IHA in a patient who presented with gradually progressing anemia. To our knowledge, alectinib-induced IHA has not been reported before; in addition, in this report, we present the first case in a patient with lung adenocarcinoma.

In conclusion, we should focus on the clinical course of anemia and hemolysis progression before and after administration of the offending drug in patients with suspected DIIHA. This is especially applicable in DAT-negative cases where clinical suspicion is high.

\section{The authors state that they have no Conflict of Interest (COI).}

\section{References}

1. Garratty G. Immune hemolytic anemia associated with drug therapy. Blood Rev 24: 143-150, 2010.

2. Chapman AM, Sun KY, Ruestow P, Cowan DM, Madl AK. Lung cancer mutation profile of EGFR, ALK, and KRAS: meta-analysis and comparison of never and ever smokers. Lung Cancer 102: 122-134, 2016.

3. Thai AA, Solomon BJ. Treatment of ALK-positive nonsmall cell lung cancer: recent advances. Curr Opin Oncol 30: 84-91, 2018.

4. Open-label randomized PhaseIII Study of the Efficacy and Safety of CH5424802(AF802) in ALK-Positive Advanced or Recurrent Non-Small Cell Lung Cancer with Crizotinib control [Internet]. [cited 2019 Nov 19]. Available from: http://image.packageinsert.jp/ pdf.php? mode $=1 \&$ yjcode $=4291032 \mathrm{M} 1029$

5. Dhaliwal G, Cornett PA, Tierney LM Jr. Hemolytic anemia. Am Fam Physician 69: 2599-2606, 2004.

6. Khan U, Ali F, Khurram MS, Zaka A, Hadid T. Immunotherapyassociated autoimmune hemolytic anemia. J Immunother Cancer 5: 15, 2017.
7. Michel M. Warm autoimmune hemolytic anemia: advances in pathophysiology and treatment. Presse Med 43: e97-e104, 2014.

8. Barros MM, Blajchman MA, Bordin JO. Warm autoimmune hemolytic anemia: recent progress in understanding the immunobiology and the treatment. Transfus Med Rev 24: 195-210, 2010.

9. Sakhri L, Mennecier B, Quoix A. Hemolytic anemia erlotinib treatment. Rev Pneumol Clin 69: 345-350, 2013.

10. De Lavallade H, Khoder A, Hart M, et al. Tyrosine kinase inhibitors impair B-cell immune responses in CML through off-target inhibition of kinases important for cell signaling. Blood 122: 227238, 2013.

11. Anazawa R, Taniyama D, Kamata H, Miyamoto K, Sakamaki F. Two cases of rheumatoid arthritis after anticancer chemotherapy with pemetrexed for nonsmall-cell lung cancer. Ann Jpn Respir Soc 2: 269-273, 2013 (in Japanese, Abstract in English).

12. Schaer D, Geeganage S, Amaladas N, et al. The folate pathway inhibitor pemetrexed pleiotropically enhances effects of cancer immunotherapy. Clin Cancer Res 25: 7175-7188, 2019.

13. Takahashi T. Direct Antiglobulin test-negative autoimmune hemolytic anemia. Acta Haematol 140: 18-19, 2018.

14. Kamesaki T. Diagnosis and treatment of hemolytic anemia. Rinsho Ketsueki (Jpn J Clin Hematol) 56: 1894-1902, 2015.

15. Garratty G. Immune hemolytic anemia associated with negative routine serology. Semin Hematol 42: 156-164, 2005.

16. Kamesaki T, Oyamada T, Omine M, Ozawa K, Kajii E. Cut-off value of red-blood-cell-bound $\mathrm{IgG}$ for the diagnosis of Coombsnegative autoimmune hemolytic anemia. Am J Hematol 84: 98$101,2009$.

17. Parker V, Tormey CA. The direct antiglobulin test: indications, interpretation, and pitfalls. Arch Pathol Lab Med 141: 305-310, 2017.

18. Kamesaki T. Autoimmune hemolytic anemia. Nihon Naika Gakkai Zasshi (J Jpn Soc Intern Med) 103: 1599-1608, 2014 (in Japanese).

The Internal Medicine is an Open Access journal distributed under the Creative Commons Attribution-NonCommercial-NoDerivatives 4.0 International License. To view the details of this license, please visit (https://creativecommons.org/licenses/ by-nc-nd/4.0/).

(C) 2021 The Japanese Society of Internal Medicine Intern Med 60: 611-615, 2021 